\title{
Nonstationary time series prediction combined with slow feature analysis
}

\author{
G. Wang ${ }^{1}$ and X. Chen ${ }^{1,2}$ \\ ${ }^{1}$ Key Laboratory of Middle Atmosphere and Global Environment Observations, Institute of Atmospheric Physics, \\ Chinese Academy of Sciences, Beijing 100029, China \\ ${ }^{2}$ Pingtan Meteorological Bureau of Fujian Province, Pingtan 350400, China
}

Correspondence to: G. Wang (wgl@ mail.iap.ac.cn)

Received: 13 October 2014 - Published in Nonlin. Processes Geophys. Discuss.: 26 January 2015

Revised: 3 April 2015 - Accepted: 16 June 2015 - Published: 10 July 2015

\begin{abstract}
Almost all climate time series have some degree of nonstationarity due to external driving forces perturbing the observed system. Therefore, these external driving forces should be taken into account when constructing the climate dynamics. This paper presents a new technique of obtaining the driving forces of a time series from the slow feature analysis (SFA) approach, and then introduces them into a predictive model to predict nonstationary time series. The basic theory of the technique is to consider the driving forces as state variables and to incorporate them into the predictive model. Experiments using a modified logistic time series and winter ozone data in Arosa, Switzerland, were conducted to test the model. The results showed improved prediction skills.
\end{abstract}

\section{Introduction}

Many previous studies have concluded that the climate system is essentially nonstationary (Trenberth, 1990; Tsonis, 1996; Yang and Zhou, 2005; Boucharel et al., 2009). However, the lack of any general theory for predicting nonstationary processes has become one of the main barriers in the field of climate prediction. To unravel this issue, in recent years, increasing effort has been devoted to devising methods to analyze and predict nonstationary time series (e.g., Hegger et al., 2000; Verdes et al., 2000; Wan et al., 2005; Wang and Yang, 2005; Yang et al., 2010). The most used method in such studies was to remove or reduce the nonstationarity of the predicted system using some mathematical techniques, thereby improving the prediction skills.
The nonstationarity exists due to the fact that the driving forces that perturb the observed system change with time (Manuca and Savit, 1996). Consequently, the most effective way to remove the nonstationarity may be to incorporate all the driving forces into the constructed dynamical system, and to consider them as the state variables of that system when establishing a prediction equation within a general circulation model (GCM). Based on this principle, lately a data-driven modeling path that is compatible with a GCM was proposed to predict several artificial nonstationary time series with known external forces. It has achieved success in improving predictions when driving forces were included in some ideal or climate systems, such as the Lorenz system, a logistic model, or global temperature over seasonal timescales including the North Atlantic Oscillation (NAO), the Pacific Decadal Oscillation (PDO), the El Niño-Southern Oscillation (ENSO), and the North Pacific Index (NPI) variability (Wang et al., 2012, 2013). However, a disadvantage of this technique is that it can not differentiate between the assumed driving forces from the predictive model. Therefore, in the present study, we considered the extraction of driving forces from the time series itself and established a predictive model by incorporating the constructed driving forces. As a result, the extraction of driving forces became the focus of this study.

Wiscott (2003) developed a technique called slow feature analysis (SFA) to extract driving forces from time series. This technique has been applied to nonstationary time series with some success (Wiskott, 2003; Berkes and Wiskott, 2005; Gunturkun, 2010; Konen and Koch, 2011). In this paper, we used SFA to construct the driving forces from a test- 
ing time series, and then established a predictive model that incorporated the driving forces. The paper is organized as follows: a brief description of the predictive technique is presented in Sect. 2. In Sect. 3, results are reported from applying the approach to a modified logistic time series and the total ozone data of Arosa, Switzerland. A summary is provided in Sect. 4.

\section{Methodology}

SFA is a method that extracts slowly varying driving forces from a quickly varying nonstationary time series. In this section, we provide a brief overview of SFA and its application for the extraction and construction of the driving forces from the time series. The details of SFA are presented in Wiscott (2003), but the basic steps of the technique are provided here for convenience and completeness. Let us assume that we have a single variable time series $\{x(t)\}_{t=1,2, \ldots n}$ from a dynamical system.

1. Embed the above time series into an m-dimensional space (also named the length of the $\mathrm{m}$ window), a phase trajectory in the m-dimensional space denoted as

$$
\begin{aligned}
& X(t)=\{x(t), x(t-1), \ldots, x(t-(m-1))\}_{t=1, \ldots, N} \text { or } \\
& X(t)=\left\{x_{1}(t), x_{2}(t), \ldots, x_{m}(t)\right\}_{t=1, \ldots, N}
\end{aligned}
$$

where $N=n-m+1$.

2. Generate an expanded signal $H(t)$ for a quadratic expansion; all monomials of degree 1 and 2 including mixed terms are used:

$$
\begin{aligned}
& H(t)=\left\{x_{1}(t), \ldots, x_{m}(t), x_{1}^{2}(t), \ldots, x_{1}(t) x_{m}\right. \\
& \left.(t), \ldots, x_{m-1}^{2}(t), x_{m-1}(t) x_{m}(t), x_{m}^{2}(t)\right\}_{t=1, \ldots, N},
\end{aligned}
$$

where $H(t)$ is a $k \times N$ matrix and $k=m+m(m+1) / 2$. Simplify (2) as

$$
H(t)=\left\{h_{1}(t), h_{2}(t) \ldots, h_{k}(t)\right\}_{t=1, \ldots, N} .
$$

The general objective of SFA is to extract slowly varying features from the time series $\{x(t)\}_{t=1,2, \ldots n}$, in other words, to find a set of coefficients, $W^{*}=$ $\left(w_{1}^{*}, w_{2}^{*}, \ldots, w_{K}^{*}\right)$, to make the output signal $y^{*}(t)=$ $W^{*} \cdot H(t)$ satisfy

$$
\left(\dot{y}^{*} \dot{y}^{* T}\right)=\min _{k}\left\{\left(\dot{y}_{k} \dot{y}_{k}^{T}\right)\right\} .
$$

Here, $\dot{y}_{k}$ is a first-order derivative, calculated by $\Delta y_{k}\left(t_{i}\right)=y_{k}\left(t_{i+1}\right)-y_{k}\left(t_{i}\right)$.

3. Normalize the expanded signal $\mathrm{H}(\mathrm{t})$ by an affine transformation to generate $H^{\prime}(t)$ with zero mean and a unit covariance matrix:

$$
H^{\prime}(t)=\left\{h_{1}^{\prime}(t), h_{2}^{\prime}(t) \ldots, h_{k}^{\prime}(t)\right\}_{t=1, \ldots, N},
$$

$$
\begin{aligned}
& \text { where } \bar{h}_{j}^{\prime}=0, h^{\prime}{ }_{j} h_{j}^{\prime}{ }_{j}=1, h^{\prime}{ }_{j}(t)=\left(h_{j}(t)-\bar{h}_{j}\right) / S \text { and } \\
& S=\frac{1}{k} \sqrt{\sum_{j=1}^{k}\left(h_{j}(t)-\bar{h}\right)^{2}} .
\end{aligned}
$$

4. By means of the Schmidt algorithm, the function space Eq. (5) is orthogonalized as

$$
\begin{aligned}
& z_{1}(t)=h_{1}^{\prime}(t), \\
& z_{j}(t)=h_{j}^{\prime}(t)-\sum_{i=1}^{j-1} \frac{h_{i+1}^{\prime}(t) \cdot z_{i}(t)}{\left\|z_{i}\right\|} z_{i}(t) \quad(j=2, \ldots, K),
\end{aligned}
$$

which is also denoted as $Z(t)=$ $\left\{z_{1}(t), z_{2}(t) \ldots, z_{k}(t)\right\}_{t=1, \ldots N}$. Here, $z_{i}(t) \cdot z_{j}(t)=0 \mathrm{i} \neq \mathrm{j}$ and it guarantees that every variable of the output is uncorrelated.

5. Establish the covariance matrix of $Z(t)$, denoted as $B=\left(\dot{Z} \dot{Z}^{T}\right)_{K \times K}$. The $\boldsymbol{k}$ eigenvectors with the smallest eigenvalues, $\lambda_{K}$, yield the normalized weight vectors with $\lambda_{1} \leq \lambda_{2} \leq \ldots \leq \lambda_{k}$, which can be easily found by principal component analysis. The smallest eigenvalue, $\lambda_{1}$, corresponding to the eigenvector $W_{1}$, can satisfy Eq. (4), which represents the weight coefficient of the slowest varying component. Here, $W_{1}$ has a free scale factor (presented as $r$ ), and then the slowest varying variable, or the driving forcing, can be obtained by the following equation:

$y_{1}(t)=r W_{1} \cdot Z(t)+c$,

where $c$ is a given constant and $\left\{y_{1}(t)\right\}$ is the output signal of the slowest driving force obtained by Eq. (7).

In this study, the SFA was tested on a logistic map

$s_{t+1}=\mu_{t} s_{t}\left(1-s_{t}\right)$

with a given driving force parameter

$$
\mu_{t}=3.5-0.45 \cos (3 \pi t / 1600) \exp (-t / 2500) .
$$

To test the ability to construct the driving force from this modified logistic map, we took a time series that consists of 5000 data points from this map. Applying the SFA algorithm to this time series with the embedded dimension chosen as 3, we constructed the driving force shown in Fig. 1, in which the dotted line represents the true driving force given by Eq. (9) and the solid line the driving force constructed by the SFA approach. There is good agreement between the constructed and true driving forces, with a correlation coefficient of 0.998. This suggests that SFA was able to extract the driving force from the observed time series in an unsupervised manner.

So far we have two time series: one is the original time series $\{x(t)\}$; the other is the slowest driving force $\left\{y_{1}(t)\right\}$. Next, we demonstrate how to establish a predictive model 


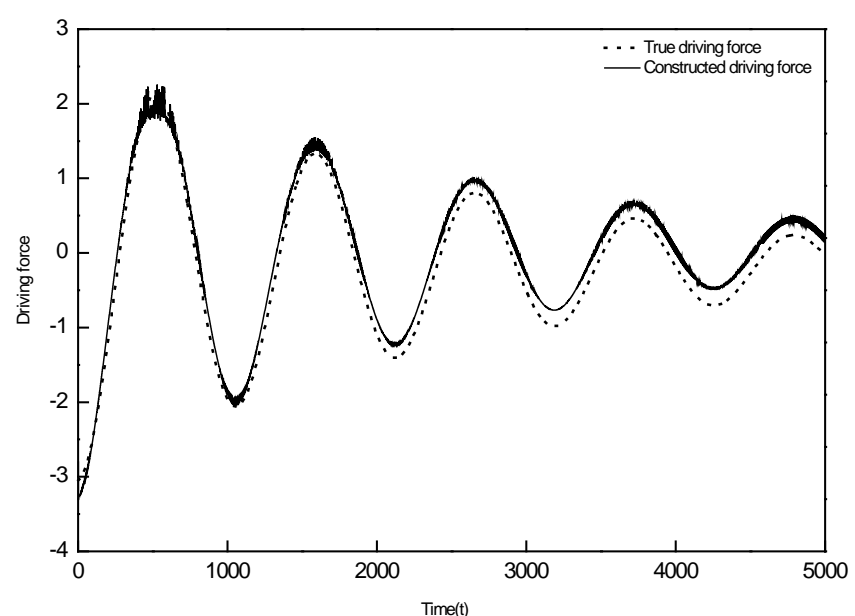

Figure 1. The true and constructed driving forces.

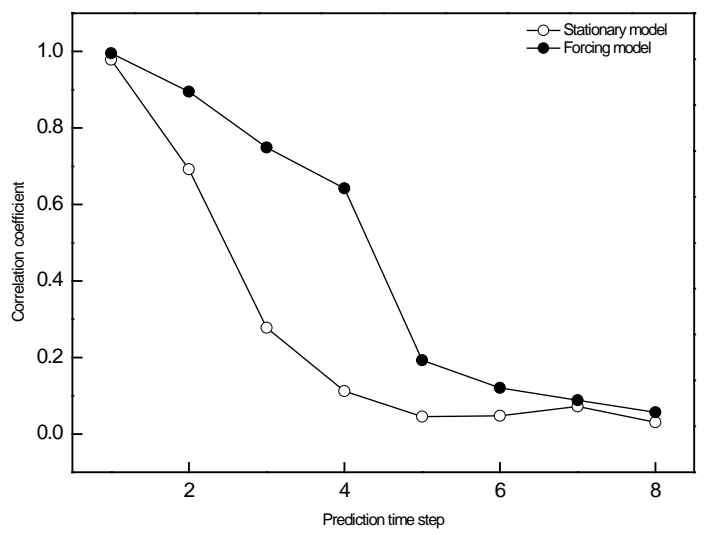

Figure 2. The comparison of prediction skills between models combined with or without driving force.

that includes the driving force constructed by the SFA procedure described above. We present the basic principle to build the prediction model; for convenience, we assume a nonstationary process is composed of two series, $\{x(t)\}_{t=1,2, \ldots n}$ and $\left\{y_{1}(t)\right\}$, with the former being the state variable time series and the latter the constructed external driving force obtained through the SFA approach. The two time series were embedded in an $m_{1}+m_{2}$ dimensional phase space with a selected time lag $\tau$. The constructed phase trajectory using the embedding theorem of Takens (1981) is shown as

$\boldsymbol{E}(t)=\left\{x(t), x(t-\tau), \ldots, x\left(t-\left(m_{1}-1\right) \tau\right) ;\right.$

$\left.y_{1}(t), y_{1}(t-\tau), \ldots, y_{1}\left(t-\left(m_{2}-1\right) \tau\right)\right\}_{t=1,2, \ldots, N}$.

Here, $m_{1}$ and $m_{2}$ are the given embedding dimensions for $\{x(t)\}$ and $\left\{y_{1}(t)\right\}$, respectively, and $N=n-\left(\max \left(m_{1}\right.\right.$, $\left.\left.m_{2}\right)-1\right) \tau$ is the number of phase points on the trajectory.

Based on this trajectory, a predictive model to predict the future state of the system can be established as

$x(t+p)=\hat{f}_{p}\left(\boldsymbol{x}(t) ; \boldsymbol{y}_{1}(t)\right)+\varepsilon(t)$.

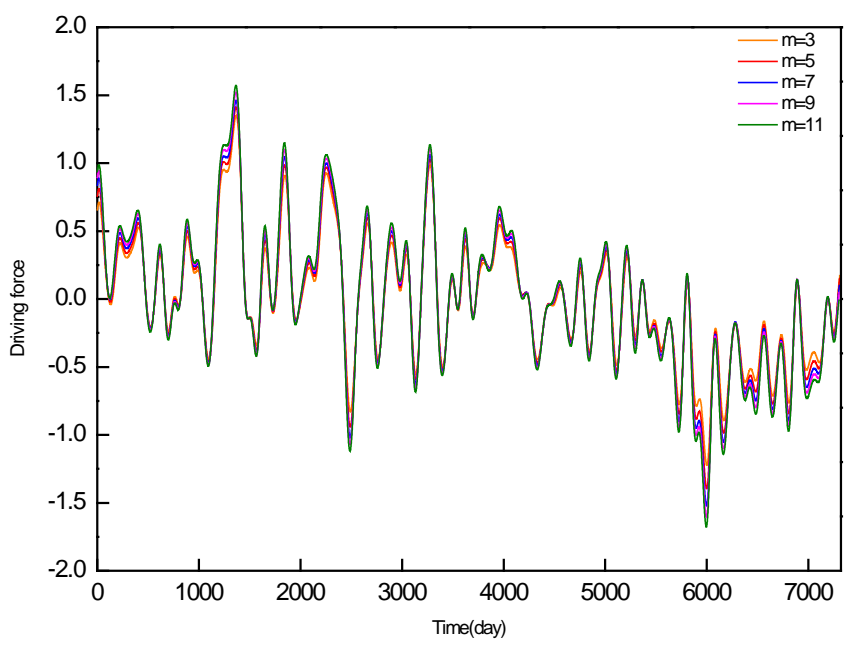

Figure 3. The slowest driving force with different embedding dimensions for total ozone data.

Where $p$ is the prediction time step (considered as 1 in the present study), $\varepsilon(t)$ is the fitting error, and $\hat{f}$ is assumed to be a quadratic polynomial in this study. The Takens embedding theorem is only appropriate for an autonomous dynamical system; therefore, we followed the method of Stark (1999) to embed the driving forces in the same state space for a nonstationary system. The next task is to find the cost function

$\eta=\sum_{t=1}^{N}\left[f\left(x(t), y_{1}(t)\right)-x(t+1)\right]^{2}$,

when it reaches its minimum value. For more details, refer to the studies of Farmer and Sidorowich (1987) and Casdagli (1989).

\section{Experiments}

We applied the prediction technique described above to perform some prediction experiments using several given nonstationary time series. The experiment presented in Sect. 3.1 was performed with data from the modified logistic model given above.

\subsection{Prediction experiments for ideal time series}

The prediction experiments were based on 5000 data points from the above-verified logistic map (Eq. 8) with the assumed driving force (Eq. 9). The first 4800 data points were applied to establish the predictive model, and the remaining 200 data points were used to test the prediction and estimate the correlation coefficient between the actual and predicted values as a function of the prediction time step. The embedding dimension of the verified logistic time series, namely $m_{1}$, took values from 2 to 3 , and the embedding dimension of the driving force time series, namely $m_{2}$, was set to either 0 (the driving force was not taken into account, and is 
Table 1. RMSE comparison of the prediction experiments (unit: Dobson units).

\begin{tabular}{lllllllllll}
\hline & 1 & 2 & 3 & 4 & 5 & 6 & 7 & 8 & 9 & 10 \\
\hline Stationary model & 0.80 & 0.88 & 0.90 & 0.94 & 0.96 & 0.99 & 1.03 & 1.02 & 1.04 & 1.05 \\
\hline Forcing model & 0.62 & 0.55 & 0.62 & 0.74 & 0.87 & 0.93 & 0.97 & 0.98 & 1.01 & 1.01 \\
\hline
\end{tabular}

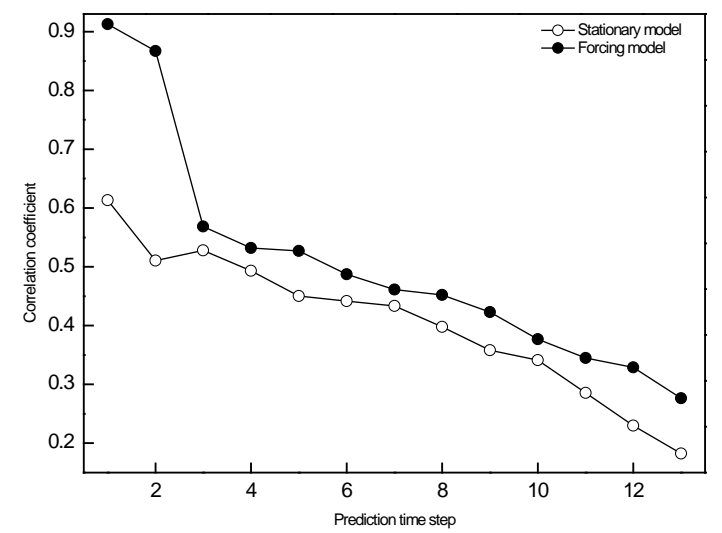

Figure 4. The comparison of prediction skills between models combined with or without driving force.

referred to as the "stationary model" hereinafter) or 1 (the driving force extracted from the verified logistic map by SFA was taken into account, and is referred to as the "forcing model" hereinafter). The time lag $\tau$ was always set to 1 . Figure 2 shows the prediction skill with and without the influence of the driving force, which was constructed by the SFA approach. The forcing model excelled over the stationary model. In particular, at the fourth prediction step, the correlation coefficients were below 0.2 in the stationary model compared to above 0.6 in the forcing model. The average correlation across the prediction time steps was improved, indicating that introducing the driving force extracted through the SFA approach into the prediction model can yield a significant improvement in accuracy.

\subsection{Prediction experiment for total ozone}

Many studies have sought to explain the variables involved in ozone dynamics, such as the Quasi-Biennial Oscillation (QBO), the 11-year solar cycle, volcanic eruptions, the El Niño-Southern Oscillation (ENSO), and the North Atlantic Oscillation (NAO) (e.g., Brasseur and Granier, 1992; Hood, 1997; Schmidt et al., 2010; Rieder et al., 2010). In this paper, we focused on prediction experiments with total ozone data. The total ozone data were from Arosa, Switzerland, and were the world's longest total ozone record. Homogenized total ozone data from 1927 to 2007 were obtained from the World Ozone and Ultraviolet Radiation Data Centre (WOUDC; http://www.woudc.org).

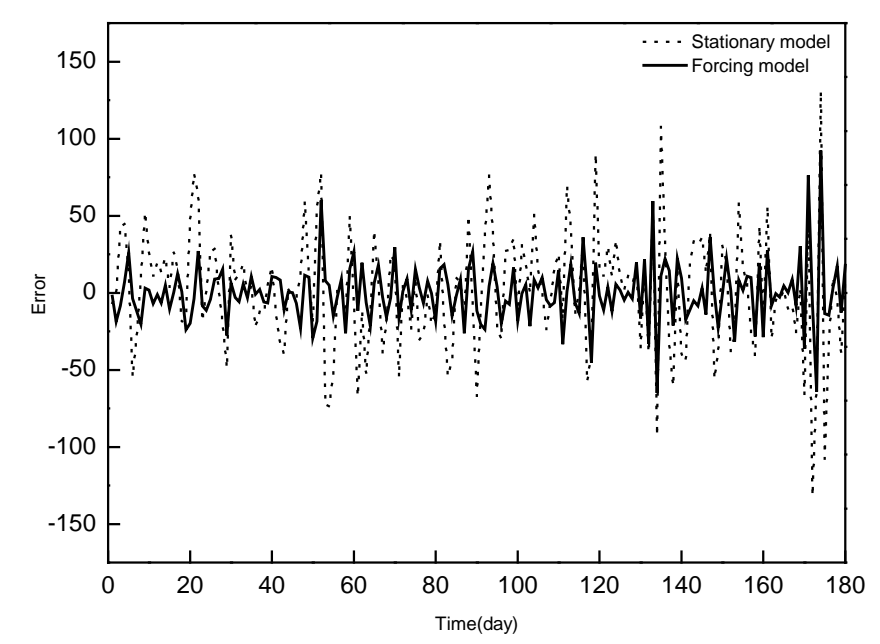

Figure 5. Errors (Dobson units) at prediction steps with or without forcing input.

By using the SFA technique on Arosa's daily total ozone data in winter (from January to March) for the period 1927 to 2007, we obtained the first output of the driving force $\left\{y_{1}\right\}$ when the embedding dimension was chosen as $3,5,7,9$, and 11, respectively (shown in Fig. 3). Note that the result did not change significantly with different embedding dimension values.

We established a prediction model for winter ozone data by incorporating the driving force constructed by SFA. The prediction was based on 7305 data points. Out of the 7305 data points, the first 7125 data points were used to build the predictive model, and the remaining 180 data points were used to test the prediction using root-mean-square error (RMSE) and the correlation coefficient between observed and predicted values. The time lag $\tau$ was taken to be 1 , the embedding dimension of the total ozone data $m_{1}$ took values from 3 to 5 , and the embedding dimension of the driving force time series $m_{2}$ was set to either 0 for the stationary model or 3 to 5 for the forcing model.

The experimental results for this case are listed in Table 1, and are also shown in Figs. 4 and 5. From Table 1, it can be seen that all RMSE values given by the forcing model were much lower than those given by the stationary model. Figure 4 presents the correlation coefficients between the observed and predicted values. The forcing model outperformed the stationary model, especially at the first two steps. At the first prediction step, the correlation coefficients 
reached 0.61 for the stationary model but 0.91 for the forcing model. At the eighth prediction step, the correlation coefficients were reduced to 0.39 for the stationary model, but still maintained at 0.45 for the forcing model. At the twelfth prediction step, the correlation coefficients were 0.22 and 0.33 for the stationary model and the forcing model, respectively. This has clearly shown that, when the constructed driving force is introduced, the accuracy of prediction is dramatically improved. The average correlation over the prediction time steps is improved by $50 \%$ when the driving force extracted through the SFA technique is included. Figure 5 illustrates the error between the prediction and the observation. The prediction error for every time step is lower for the forcing model than for the stationary model. All these results indicate that the inclusion of the driving force constructed by the SFA approach in the prediction model largely improves the predictive skill of winter total ozone in Arosa. Some sensitivity analysis with different training/verifying lengths does not alter this conclusion.

\section{Discussion}

In this study, we first constructed the driving forces of a time series based on the SFA approach, and then the driving forces were introduced into a predictive model. By doing so, we extend the study by Wang et al. $(2012,2013)$ and present a novel technique to predict nonstationary time series. Unlike the former works by Wang et al. $(2012,2013)$ with assumed driving forces, in this study, the driving force was extracted from original time series. The experimental results obtained from a modified logistic time series and winter ozone data in Arosa confirmed the effectiveness of the model.

The driving force construction technique based on SFA represents a progress for climate causal relations. Such an approach may provide a compatible and direct window for studying causality using external driving forces. We constructed the driving forces with SFA and then combined these driving forces to establish the predictive model. Although we found this approach was able to effectively improve the predictive ability, the constructed driving force time series still lacks physical explanation. In order to understand the real background of these, one has to further explore the physical processes behind it. One recommended method, provided by Verdes (2005), suggests using a measure called "transfer entropy" to analyze the causality; another recommended method is named "convergent cross mapping", provided by Sugihara et al. (2012), which measures causality in nonlinear dynamic systems. Work in this area is in progress and will be reported in future publications.
Acknowledgements. This research was supported by the National Natural Science Foundation of China under grant 41275087. The authors are grateful to the editor and three anonymous referees for useful comments during the discussion phase in Nonlin. Processes Geophys. Discuss.

Edited by: V. Lucarini

\section{References}

Berkes, P. and Wiskott, L.: Slow feature analysis yields a rich repertoire of complex cell properties, J. Vision, 5, 579-602, 2005.

Boucharel, J., Dewitte, B., Garel, B., and du Penhoat, Y.: ENSO's non-stationary and non-Gaussian character: the role of climate shifts, Nonlin. Processes Geophys., 16, 453-473, doi:10.5194/npg-16-453-2009, 2009.

Brasseur, G. and Granier, C.: Mount Pinatubo aerosols, chlorofluorocarbons and ozone depletion, Science, 257, 1239-1242, 1992.

Casdagli, M.: Nonlinear prediction of chaotic time series, Phys. D., 35, 335-356, 1989.

Farmer, J. D. and Sidorowich, J.: Predicting chaotic time series, Phys. Rev. E., 59, 845-848, 1989.

Gunturkun, U.: Sequential construction of driving-forces from nonlinear nonstationary dynamics, Phys. D., 239, 1095-1107, 2010.

Hegger, R., Kantz, H., Matassini, L., and Schreiber, T.: Coping with non-stationarity by over-embedding, Phys. Rev. E., 84, 4092 4101, 2000.

Hood, L.: The solar cycle variation of total ozone: Dynamical forcing in the lower stratosphere, J. Geophys. Res., 102, 1355-1370, 1997.

Konen, W. and Koch, P.: The slowness principle: SFA can detect different slow components in non-stationary time series, Int. J. Inn. Comp. Appl., 3, 3-10, 2011.

Manuca, R. and Savit, R.: Stationarity and nonstationarity in time series analysis, Phys. D., 99, 134-161, 1996.

Rieder, H. E., Staehelin, J., Maeder, J. A., Peter, T., Ribatet, M., Davison, A. C., Stübi, R., Weihs, P., and Holawe, F.: Extreme events in total ozone over Arosa - Part 2: Fingerprints of atmospheric dynamics and chemistry and effects on mean values and long-term changes, Atmos. Chem. Phys., 10, 10033-10045, doi:10.5194/acp-10-10033-2010, 2010.

Schmidt, H., Brasseur, G., and Giorgetta, M.: Solar cycle signal in a general circulation and chemistry model with internally generated quasi-biennial oscillation, J Geophys Res., 115, D00I14, doi:10.1029/2009JD012542, 2010.

Stark. J.: Delay embeddings for forced systems: deterministic forcing, J. Nonl. Sci., 9, 255-332, 1999.

Sugihara, G., May, R., Ye, H., Hsieh, C., Deyle, E., Fogarty, M., and Munch, S.: Detecting causality in complex ecosystems, Science, 338, 496-500, doi:10.1126/science.1227079, 2012.

Takens, F.: Detecting Strange Attractors in Turbulence, Dynamical Systems and Turbulence, Heidelberg, Springer-Verlag, 366-381, 1981.

Trenberth, K. E.: Recent observed inter-decadal climate changes in the northern hemisphere, B. Am. Meteor. Soc., 7, 988-993, 1990.

Tsonis, A. A.: Widespread increases in low-frequency variability of precipitation over the past century, Nature, 382, 700-702, 1996. 
Verdes, P. F.: Assessing causality from multivariate time series, Phys. Rev. E., 72, 026222, doi:10.1103/PhysRevE.72.026222, 2005.

Verdes, P. F., Parodi, M. A., Granitto, P. M., Navone, H. D., Piacentini, R. D., and Ceccatto, H. A.: Predictions of the maximum amplitude for solar cycle 23 and its subsequent behavior using nonlinear methods, Sol. Phys., 191, 419-425, 2000.

Wan, S., Feng, G., Zhou, G., Wan, B., Qin, M., and Xu, X.: Extracting useful information from the observations for the prediction based on EMD method, Ac. Meteorol. Sin., 63, 516-525, 2005.

Wang, G. and Yang, P.: A compound constructed prediction model for nonstationary climate process, Inter. J. Climatol., 25, 12651277, 2005.

Wang, G., Yang, P., Zhou, X., Swanson, K., and Tsonis, A.: Directional influences on global temperature prediction, Geophys. Res. Lett., 39, L13704, doi:10.1029/2012GL052149, 2012.
Wang, G., Yang, P., and Zhou, X.: Nonstationary time series prediction by incorporating external forces, Adv. Atmos. Sci., 30, 1601-1607, 2013.

Wiskott, L.: Estimating driving forces of nonstationary time series with slow feature analysis, arXiv.org e-Print archive, http://arxiv. org/abs/cond-mat/0312317/ (last access: 12 December 2003), 2003.

Yang, P. and Zhou, X.: On nonstationary behaviors and prediction theory of climate systems, Ac. Meteorol. Sin., 63, 556-570, 2005

Yang, P., Wang, G., Bian, J., and Zhou, X.: The prediction of nonstationary climate series based on empirical mode decomposition, Adv. Atmos. Sci., 27, 845-854, doi:10.1007/s00376-0099128-x, 2010. 